\title{
SOBRE EL CONCEPTO DE CONSTITUCIÓN ${ }^{1}$
}

\section{Riccardo GUASTINI ${ }^{2}$}

RESUMEN: En el artículo se examinan sistemáticamente los diferentes usos del concepto de "Constitución" en el lenguaje jurídico y político. Para ello, sin intentar un estudio exhaustivo, se revisan los cuatro sentidos que el autor considera principales, a saber, el de Constitución como un ordenamiento político de tipo liberal; como el conjunto de normas "fundamentales"; como un documento normativo que tiene ese nombre, y como un texto normativo dotado de ciertas características "formales" propio de un régimen jurídico específico. Al examinar sucesivamente estas cuestiones, el autor expone los diferentes sentidos del concepto de Constitución y aborda algunos de los problemas de la teoría constitucional, como el de la diferencia entre Constitución y otras leyes, el contenido de la Constitución, la Constitución como fuente y los poderes constituyentes y constituidos.
ABSTRACT: This article systematically examines the different uses of the concept of "Constitution" in legal and political language. For this purpose, without attempting an exhaustive study, an examination is made of the four senses considered most important by the author, i.e., that of Constitution as a political ordinance of a liberal nature; as a group of "fundamental" norms; as a normative document bearing this name, and as a normative text possessing certain "formal" characteristics appropriate for a specific legal system. On examining these matters one by one, the author puts forward different senses of the concept of Constitution and takes into consideration some of the problems of the constitutional theory, such as that of the difference between the Constitution and other laws, the contents of the Constitution, the constitution as a source, and constitutional and constituted powers.

1 Traducción del italiano por Miguel Carbonell (IIJ-UNAM).

2 Universidad de Génova. 


\section{VARIEDAD DE USOS DEL TÉRMINO “CONSTITUCIÓN"}

El término "Constitución" es usado en el lenguaje jurídico (y político) con una multiplicidad de significados (cada uno de los cuales presenta muy diversos matices). ${ }^{3}$ No es aquí el lugar para hacer un inventario completo. Será suficiente distinguir los cuatro significados principales, que son los siguientes:

a) en una primera acepción, "Constitución" denota todo ordenamiento político de tipo "liberal";

b) en una segunda acepción, "Constitución" denota un cierto conjunto de normas jurídicas: grosso modo, el conjunto de normas —en algún sentido fundamentales - que caracterizan e identifican todo ordenamiento;

c) en una tercera acepción, "Constitución" denota - simplemente- un documento normativo que tiene ese nombre (o un nombre equivalente);

d) en una cuarta acepción, en fin, "Constitución" denota un particular texto normativo dotado de ciertas características "formales", o sea de un peculiar régimen jurídico.

3 Sobre el concepto (o mejor, sobre los conceptos) de Constitución la literatura es obviamente inmensa. Cfr. por ejemplo Crisafulli, V., "Costituzione", Enciclopedia del novecento, I, Roma, 1975; De Vergottini, G., "Costituzione”, en Bobbio, N. y Matteucci, N. (eds.), Dizionario di politica, Turín, 1976, pp. 274 y ss. (hay traducción al castellano en la editorial Siglo XXI, de México); Burdeau, G., Traité de science politique, IV. Le statut du pouvoir dans l'état, París, 1983, cap. I; Bastid, P., L'idée de constitution, París, 1985; Modugno, F., "Il concetto di costituzione", Aspetti e tendenze del diritto costituzionale. Scritti in onore di Costantino Mortati, I, Milán, 1977, pp. 199 y ss.; id., "Costituzione (teoria generale)", Enciclopedia giuridica, X, Roma, 1988; id., Appunti per una teoria generale del diritto, Turín, 1988, pp. 57 y ss.; Zagrebelsky, G., Societá, stato, costituzione. Lezioni di dottrina dello stato, Turín, 1988, pp. 75 y ss.; Barberis, M., "Progetto per la voce 'costituzione' di una enciclopedia", Filosofia politica, 1991, pp. 351 y ss.; S. Bartole, "Costituzione (dottrine generali e diritto costituzionale"), Digesto, IV ed. (Discipline pubblicistiche), VI, Turín, 1991; Comanducci, P., Assaggi di metaetica, Turín, 1992, pp. 155 y ss.; id., "Ordine o norma? Su alcuni concetti di costituzione nel Settecento", Studi in memoria di Giovanni Tarello, I. Saggi storici, Milán, 1990, pp. 173 y ss.; Guastini, R., Quindici lezioni di diritto costituzionale, II ed., Turín, 1992, pp. 9 y ss.; Floridia, G. G., “Qu'est-ce que... la constitution?”, I viaggi di Erodoto, 18, 1992, pp. 48 y ss.; id., "'Costituzione': il nome e le cose", Analisi e diritto. Ricerche di giurisprudenza analitica, 1994, pp. 131 y ss.; Dogliani, M., Introduzione al diritto costituzionale, Bolonia, 1994, pp. 11 y ss.; Furet, F., Halévi, R., La monarchie républicaine, París, 1996, cap. I. 


\section{LA CONSTITUCIÓN COMO LÍMITE AL PODER POLÍTICO}

Para la filosofía política, el término "Constitución" es comúnmente utilizado en su sentido originario, para denotar cualquier ordenamiento estatal de tipo liberal (o, si se quiere, liberal-garantista) $;{ }^{4}$ un ordenamiento en el que la libertad de los ciudadanos en sus relaciones con el Estado esté protegida mediante oportunas técnicas de división del poder político. ${ }^{5}$

El originario concepto liberal de Constitución fue puesto en claro por el artículo 16 de la Déclaration des droits de l'homme et du citoyen (1789), que estableció lo siguiente: "Una sociedad en la que no esté asegurada la garantía de los derechos ni reconocida la división de poderes, no tiene Constitución". ${ }^{6}$

En este contexto, claro está, el término "Constitución" denota no ya una organización política cualquiera, sino una organización política liberal y garantista. La Constitución es concebida aquí como límite al poder político.

De esta forma no todo Estado está provisto de Constitución: los Estados liberales son Estados constitucionales, o sea tienen Constitución; mientras que los Estados despóticos no son Estados "constitucionales", es decir, carecen de Constitución. En este sentido del adjetivo "constitucional", un Estado puede llamarse constitucional, o provisto de Constitución, si y sólo si satisface dos condiciones (disyuntivamente necesarias

4 Matteucci, N., "Positivismo giuridico e costituzionalismo", Rivista trimestrale di diritto e procedura civile, 1963; id., Organizzazione del potere e libertá, Turín, 1976; id., "Costituzionalismo", Bobbio, N., Matteucci, N., Dizionario di politica, Turín, 1976, pp. 262 y ss.; Sartori, G., Elementi di teoria politica, Bolonia, 1987, pp. 11 y ss. (hay traducción al castellano en Alianza Editorial, Madrid, 1996).

5 Sobre el constitucionalismo liberal (y la correspondiente noción de Constitución): McIlwain, Ch. H., Constitutionalism: Ancient and Modern (1947), Bolonia, 1990, pp. 27 y ss. (hay traducción al castellano publicada por el Centro de Estudios Constitucionales, Madrid, 1991); Tarello, G., Storia della cultura giuridica moderna, I, Bolonia, 1976, pp. 22 y ss., pp. 559 y ss.; id., "Le idee giuridiche della Rivoluzione francese", S. Castignone (ed.), L'opera di Giovanni Tarello nella cultura giuridica contemporanea, Bolonia, 1989, pp. 331 y ss.; Barberis, M., Benjamin Constant. Rivoluzione, costituzione, progresso, Bolonia, 1988; pp. 93 y ss.; id., Sette studi sul liberalismo rivoluzionario, Turín, 1989, pp. 213 y ss.; Comanducci, P., "Ordine o norma? Su alcuni concetti di costituzione nel Settecento”, pp. 173 y ss.; Floridia, G. G., La costituzione dei moderni, Turín, 1991.

6 A propósito: Troper, M., "Tre esercizi di interpretazione costituzionale", Analisi e diritto. Ricerche di giurisprudenza analitica, 1990, pp. 197 y ss.; id., Pour une théorie juridique de l'Etat, París, 1994, pp. 263 y ss., pp. 317 y ss. 
y conjuntivamente suficientes): a) por un lado, que estén garantizados los derechos de los ciudadanos en sus relaciones con el Estado; b) por otro, que los poderes del Estado (el Poder Legislativo, el Poder Ejecutivo o de gobierno, el Poder Judicial) estén divididos y separados (o sea que se ejerzan por órganos diversos).

Este modo de utilizar el término "Constitución” está hoy en día en desuso. No obstante, algunas expresiones todavía de uso corriente (especialmente en sede historiográfica) presuponen el concepto liberal de Constitución, y serían incomprensibles sin él. Es el caso, por ejemplo, de los términos "constitucionalismo" y "constitucionalización", , de las expresiones "monarquía constitucional", "Estado constitucional" y "gobierno constitucional" (en oposición a: "monarquía absoluta", "Estado absoluto", "gobierno absoluto").

\section{LA CONSTITUCIÓN COMO CONJUNTO DE NORMAS "FUNDAMENTALES"}

En el campo de la teoría general del derecho, el término "Constitución” es generalmente usado para designar el conjunto de las normas "fundamentales" que identifican o caracterizan cualquier ordenamiento jurídico. ${ }^{8}$

La cuestión de cuáles normas deben ser consideradas fundamentales es una cosa obviamente debatible, desde el momento que "fundamental" no denota una propiedad empírica (calificar alguna cosa como "fundamental" es, en última instancia, un juicio de valor). Normas fundamentales de un determinado ordenamiento jurídico pueden ser consideradas, según los diversos puntos de vista, por lo menos las siguientes:

1. Las normas que disciplinan la organización del Estado y el ejercicio del poder estatal (al menos en sus aspectos fundamentales: la función legislativa, la función ejecutiva y la función judicial), así como la conformación de los órganos que ejercen esos poderes (por ejemplo, las normas que disciplinan la formación del órgano legislativo); o bien,

7 Sobre el concepto de constitucionalización, cfr. Tarello, G., Storia della cultura giuridica moderna, I, pp. 22 y ss.

8 Cfr. por ejemplo Biscaretti di Ruffia, P., Diritto costituzionale, XIV ed., Napoles, 1986, p. 86. 
2. Las normas que disciplinan las relaciones entre el Estado y los ciudadanos (por ejemplo, las eventuales normas que reconocen a los ciudadanos derechos de libertad); o todavía,

3. Las normas que disciplinan la "legislación" (entendida en sentido "material", como la función de crear el derecho), o sea las normas que confieren poderes normativos, que determinan las modalidades de formación de los órganos a los que esos poderes son conferidos, que regulan los procedimientos de ejercicio de esos poderes, etcétera; ${ }^{9}$ o en fin,

4. Las normas — comúnmente, si son escritas, formuladas como declaraciones solemnes - que expresan los valores y principios que informan a todo el ordenamiento.

En general, se puede convenir en que son normas fundamentales de cualquier ordenamiento: a) las que determinan la llamada "forma de Estado"; b) las que determinan la "forma de gobierno"; y c) las que disciplinan la producción normativa. ${ }^{10}$

Es evidente que, desde este punto de vista, todo Estado tiene necesariamente su propia Constitución. Puede tratarse de una Constitución liberal o no liberal. Puede tratarse de un conjunto de normas escritas o bien consuetudinarias. Estas normas, si son escritas, pueden estar o no recogidas en un único documento. Pero, en todo caso, todos los Estados están provistos de una Constitución de cualquier tipo.

Este concepto de Constitución es característico del positivismo jurídico moderno, ${ }^{11}$ y es el que habitualmente se adopta hoy en día por los estudiosos del derecho público.

Este concepto de Constitución, a diferencia del originario ("Constitución" en sentido liberal), es un concepto políticamente "neutro": una

9 Este punto de vista es característico en Kelsen, H., General Theory of Law and State, Cambridge (Mass.), 1945, esp. pp. 124 y ss. (traducción al castellano de Eduardo García Maynez, México, UNAM, 1989); id., Théorie pure du droit (1960), París, 1962, esp. pp. 299 y ss. (trad. al castellano de Roberto J. Vernengo, México, UNAM-Porrúa, 1979).

10 Así L. Paladin, Diritto costituzionale, Padua, 1991, p. 25. La noción (en verdad un poco confusa) de "forma de Estado" se refiere en general a los diversos modos en que son organizadas las recíprocas relaciones entre los llamados elementos constitutivos del Estado (pueblo, gobierno, territorio): por ejemplo, democracia vs. autocracia, Estado unitario vs. Estado federal. La noción de forma de gobierno, a su vez, se refiere al modo en que son organizadas las relaciones recíprocas entre los órganos constitucionales (especialmente parlamento, gobierno, jefe de Estado): por ejemplo, gobierno parlamentario vs. gobierno presidencial.

11 Críticas en Matteucci, N., "Positivismo giuridico e costituzionalismo". 
Constitución es tal con independencia de su contenido político (liberal, no liberal, democrático, autocrático, etcétera).

\section{LA "MATERIA CONSTITUCIONAL"}

La Constitución entendida como conjunto de normas fundamentales (en uno u otro sentido) es llamada a su vez Constitución en sentido "sustancial" o "material". ${ }^{12} \mathrm{Al}$ concepto "material" de Constitución, entendido en este sentido específico, se conectan las nociones de "materia constitucional" y de "norma materialmente constitucional". ${ }^{13}$

- Se llaman materialmente constitucionales las normas "fundamentales" —en uno u otro sentido- de todo ordenamiento jurídico.

- Se llama materia constitucional el conjunto de objetos que son disciplinados por tales normas.

Las normas "materialmente constitucionales" pueden ser escritas o consuetudinarias. Donde existe una Constitución escrita se esperaría que esas normas estuvieran expresamente formuladas. Sin embargo, no es infrecuente que también ahí donde existe una Constitución escrita, muchas normas pacíficamente consideradas "materialmente constitucionales" no estén escritas en la Constitución (sino que estén escritas en leyes ordinarias, o también que no estén de hecho escritas, quedando implícitas, en estado latente). De la misma forma, no es infrecuente que las Constituciones incluyan también normas no "materialmente constitucionales". ${ }^{14}$

12 Cfr. por ejemplo Romano, S., Principii di diritto costituzionale generale, reimpr. de la II edic., Milán, 1947, pp. 1 y ss. En este contexto, la expresión "Constitución material" (o "sustancial”) tiene un sentido un poco diverso de otros más difundidos sobre los que volveremos de inmediato.

13 Cfr. por ejemplo De Vergottini, G., Diritto costituzionale comparato, Padua, 1991, pp. 154 y ss. (y la bibliografía citada); Cicconetti, S. M., La revisione della costituzione, Padua, 1972, pp. 54 y ss.

14 “También lo que no esté contenido en la Constitución formal puede ser materialmente constitucional y... no todo lo que está contenido en la Constitución formal es también materialmente constitucional” (Cicconetti, S. M., La revisione della costituzione, p. 4). 


\section{LA CONSTITUCIÓN COMO “CÓDIGO” DE LA MATERIA CONSTITUCIONAL}

En el lenguaje común, como también para la de teoría de las fuentes, el término "Constitución" es comúnmente utilizado para designar un específico documento normativo $-\mathrm{O}$ sea un texto, formulado en una lengua natural, y expresivo de normas (jurídicas)— que formula y recoge, si no todas, al menos la mayor parte de las normas materialmente constitucionales de un ordenamiento determinado. ${ }^{15}$ En esta acepción, en suma, la Constitución es una suerte de "código" (si bien el término "código" no se suele utilizar en este contexto) de la materia constitucional. Al igual que es verdad que es raro que todas las normas constitucionales contenidas en una Constitución sean "materialmente constitucionales", también es raro que la Constitución agote toda la "materia constitucional".

El "código" constitucional, allí donde existe, es un texto que se distingue de otros documentos normativos (en particular de las "leyes" comunes) al menos por las siguientes características.

En primer lugar, la Constitución se distingue de otros textos normativos en virtud de su nombre propio: el nombre de "Constitución" (o en otros ordenamientos: "carta", "carta constitucional", "estatuto", "ley fundamental" y similares), que corresponde a veces a una peculiar formulación: normalmente las Constituciones o una de sus partes (especialmente los eventuales preámbulos) están redactadas en un lenguaje solemne, destinado a subrayar la importancia política del documento.

El nombre "Constitución" — se puede observar - individualiza, en todo ordenamiento, no ya un tipo (una clase) de textos, sino un singular documento normativo. En otras palabras, desde el punto de vista sincrónico, cualquier ordenamiento jurídico incluye - junto a una multiplicidad de leyes, de reglamentos, etcétera - no ya también una pluralidad de Constituciones, sino una, y una sola, Constitución.

15 Por otro lado, el nombre "Constitución", fatalmente, evoca los otros significados del término, a los que ya nos hemos referido. De modo que, por un lado, la "Constitución" se presenta como fuente que recoge, si no todas, al menos algunas de las normas fundamentales del ordenamiento (en el sentido que se ha indicado); por otro lado, la existencia misma de una "Constitución" sugiere a veces engañosamente que el ordenamiento de que se trata es un ordenamiento de tipo liberal. 
En segundo lugar, la Constitución se distingue de las otras fuentes del derecho en virtud de su contenido característico. Como se ha dicho, es raro que todas las normas contenidas en una Constitución sean "materialmente constitucionales", y es igualmente raro que la Constitución agote la "materia constitucional". No obstante, las Constituciones tienen en gran medida un contenido "materialmente constitucional", en el sentido que se ha apuntado.

Normalmente, las Constituciones incluyen: ${ }^{16}$

a) normas que confieren derechos de libertad a los ciudadanos, disciplinando de esa forma las relaciones entre los ciudadanos y el poder político;

b) normas sobre la legislación y más en general normas que confieren poderes a los órganos del Estado, disciplinando así la organización del poder político mismo.

Muchas Constituciones contemporáneas, además, incluyen también una multiplicidad de normas "de principio" o de normas "programáticas". Unas contienen los valores y principios que informan - $\mathrm{O}$ al menos eso se supone- a todo el ordenamiento jurídico. Las otras recomiendan al legislador (y eventualmente a la administración pública) perseguir programas de reforma económica y/o social.

En tercer lugar, la Constitución se distingue de las otras fuentes del derecho en virtud de sus destinatarios típicos: si no todas, casi todas las normas constitucionales se refieren no ya a los ciudadanos particulares, y ni siquiera a los órganos jurisdiccionales comunes, sino a los órganos constitucionales supremos (como: el jefe de Estado, las cámaras, el gobierno, la Corte Constitucional, etcétera).

Se observa que cuando se emplea el vocablo "Constitución" en el sentido de código constitucional, no se puede decir que todo Estado esté necesariamente provisto de una Constitución. Es sin embargo verdad que la gran mayoría de los Estados contemporáneos poseen un código constitucional. Pero ningún Estado del "antiguo régimen” lo poseía de hecho. En nuestra época son raros o rarísimos los Estados que no lo poseen: se suele citar el ejemplo de Gran Bretaña, cuyo derecho constitucional es en gran parte consuetudinario (y por tanto no codificado). De todas for-

16 Tarello, G., Storia della cultura giuridica moderna, I, pp. 607 y ss. Cfr. también Kelsen, H., General Theory of Law and State, 260 y ss.; De Vergottini, G., Diritto costituzionale comparato, pp. 153 y ss. 
mas, no existen razones lógicas que excluyan la existencia de un Estado desprovisto de código constitucional.

\section{LA CONSTITUCIÓN COMO FUENTE DIFERENCIADA}

En fin, el término "Constitución" es comúnmente utilizado para referirse a una fuente del derecho a un documento normativo que se diferencia de cualquier otra fuente por algunas características "formales".

En primer lugar, la Constitución se distingue de otros textos normativos en virtud de su procedimiento de formación, que es diverso de todas las demás fuentes del derecho. Muchas Constituciones, por ejemplo, son fruto de la elaboración y aprobación por parte de una asamblea "constituyente" elegida para ese propósito; otras traen legitimidad de un reférendum popular; otras sin embargo, son fruto de una decisión unilateral del soberano (Constituciones llamadas "otorgadas", es decir, dadas generosamente por el soberano a "su" pueblo); etcétera. ${ }^{17}$

En segundo lugar, la Constitución se distingue algunas veces (no siempre) de otras fuentes del derecho - en particular- de las leyes en virtud de un régimen jurídico especial, de una "fuerza" peculiar, que la pone "por encima" de las leyes (y de cualquier otra fuente). Gozan de un régimen jurídico especial; en este sentido, las Constituciones que no pueden ser abrogadas, derogadas o modificadas por las leyes, en las que el procedimiento de revisión constitucional es diverso del —más complejo que el- procedimiento legislativo ordinario. Tales Constituciones se llaman rígidas.

\section{LA CONSTITUCIÓN Y LAS (OTRAS) LEYES}

Las Constituciones (escritas) son "leyes" en sentido genérico, o sea textos normativos: documentos que expresan normas (jurídicas). Pero ¿qué distingue a una Constitución de las demás leyes? Esta pregunta admite diversas respuestas, cada una de las cuales supone un diverso concepto de Constitución.

i) Se puede opinar ante todo que la Constitución se distingue de las otras leyes en virtud de su función característica: la función de las Cons-

17 De Vergottini, G., Diritto costituzionale comparato, pp. 138 y ss. 
tituciones es limitar el poder político. Este punto de vista está conectado al concepto liberal de Constitución.

ii) Se puede opinar además que la Constitución se distingue de las otras leyes en virtud de su contenido: contenido típico de las Constituciones es la distribución de los poderes en el seno del aparato estatal y la disciplina de las relaciones entre el Estado y los ciudadanos. Este punto de vista está conectado al concepto de Constitución como conjunto de normas "fundamentales".

iii) Se puede afirmar, en fin, que la Constitución se distingue de las otras leyes en virtud no de su contenido, sino prescindiendo de él, es decir, en virtud de su "forma". ¿En qué sentido?

a) En un primer sentido (débil), "forma" denota el aspecto exterior de las Constituciones: son Constituciones todos y solamente aquellos documentos normativos que tienen ese nombre (cualquiera que sea su contenido normativo). De este modo, las Constituciones consuetudinarias no son "verdaderas" Constituciones.

b) En un segundo sentido (fuerte), "forma" denota el régimen jurídico o la "fuerza" de algunas Constituciones: son Constituciones todos y solamente aquellos documentos normativos que no pueden ser abrogados, derogados o modificados por (otras) leyes. De este modo, las Constituciones flexibles no son, propiamente hablando, "Constituciones".

\section{LA CONSTITUCIÓN COMO FUENTE: ¿EN QUÉ SENTIDO?}

En general, las Constituciones escritas - $\mathrm{o}$ Constituciones en sentido formal- son fuentes del derecho. ${ }^{18}$ ¿En qué sentido? Esta tesis puede ser entendida en no menos de tres modos diversos.

En un primer sentido, se puede decir que la Constitución es fuente del derecho por entender, simplemente, que los enunciados constitucionales expresan normas (ya sea normas en sentido genérico, ya sea normas en sentido estricto, o sea mandatos), las cuales disciplinan - cuando menos - la organización del Estado y las relaciones entre el Estado y los ciudadanos.

18 Que la Constitución sea fuente del derecho no puede decirse de las Constituciones consuetudinarias: una Constitución consuetudinaria, de hecho, es un conjunto de normas, y no una fuente de normas (fuente de normas es la costumbre de la que la Constitución nace, no la Constitución misma). 
Este primer modo de entender la tesis en examen es, prima facie, absolutamente obvio y no problemático.

En un segundo sentido, se puede decir que la Constitución es fuente del derecho por entender que las normas constitucionales -al menos bajo un régimen de Constitución rígida - son idóneas:

a) para abrogar y/o invalidar (se trata, en este caso, de invalidez sobrevenida) normas anteriores de rango subconstitucional materialmente incompatibles con ella;

b) para invalidar normas sucesivas de rango subconstitucional formalmente disconformes o materialmente incompatibles con ella.

Este segundo modo de entender la tesis en cuestión es menos obvio desde el momento que, en Italia la doctrina y la jurisprudencia, al menos en relación con algunas normas constitucionales, han sostenido una opinión contraria. ${ }^{19}$

En un tercer sentido, se puede decir que la Constitución es fuente del derecho por entender que las normas constitucionales son idóneas para disciplinar directamente no sólo la organización estatal y las relaciones entre el Estado y los ciudadanos, sino también las relaciones entre particulares, y son por tanto susceptibles de aplicación jurisdiccional por parte de cualquier juez (y no solamente por parte del juez constitucional). ${ }^{20}$

También este tercer modo de entender la tesis en examen no es obvio, ya sea porque está en contraste con la concepción clásica de la Constitución (como límite al poder político), ya sea porque, más en general, está conectado a un cierto modo de interpretar los textos constitucionales.

19 Se alude a la distinción entre normas "preceptivas" y normas "programáticas" y/o "de principio".

20 Cfr. Zagrebelsky, G., Manuale di diritto costituzionale, I, reimpresión actualizada, Turín, 1991, p. 105: "Donde la estructura de la norma constitucional es suficientemente completa para poder valer como regla para casos concretos, debe ser utilizada directamente por todos los sujetos del ordenamiento jurídico, ya sean los jueces, la administración pública o los particulares. La Constitución es en suma fuente directa de posiciones subjetivas para los sujetos del ordenamiento, en todo tipo de relaciones en que puedan entrar... Hoy la Constitución se dirige también, directamente, a las relaciones entre los individuos y a las relaciones sociales. Por eso las normas constitucionales pueden ser invocadas, cuando sea posible, como reglas, por ejemplo, para las relaciones familiares, en las relaciones en las empresas, en las asociaciones y así por el estilo". Cfr. también Barile, P., La costituzione come norma giuridica; Dogliani, M., Interpretazioni della costituzione, Milán, 1982; García de Enterría, E., La Constitución como norma y el Tribunal Constitucional, 3a. ed., Madrid, 1985. 


\section{PODER CONSTITUYENTE}

La noción de poder constituyente ${ }^{21}$ - si es oportunamente depurada de incrustaciones ideológicas - se define, simplemente, por oposición a la de poder constituido. ${ }^{22}$

Se llama "constituido" a todo poder "legal", es decir, conferido y disciplinado por normas positivas vigentes (y ejercido de conformidad con ellas). Las normas que provienen de un poder constituido encuentran su fundamento de validez en las normas sobre la producción jurídica vigentes.

Se llama por el contrario "constituyente" al poder de instaurar una "primera" Constitución. ${ }^{23}$

Llamo "primera Constitución" a toda Constitución que no encuentre su fundamento de legitimidad en una Constitución precedente. ${ }^{24}$ Una primera Constitución es en suma una Constitución emanada extra ordinem - fruto de una revolución - y por tanto privada de fundamento de validez en normas (las eventuales normas sobre la producción constitucional) propias del ordenamiento constitucional precedente. ${ }^{25}$

Dicho esto, sin embargo, hay que agregar que detrás de esta aparente simplicidad, la noción de poder constituyente es un poco problemática. Para aclarar al menos algunos de los problemas que involucra, puede ser conveniente destacar la siguiente cuestión.

21 A propósito cfr. Carré de Malberg, R., Contribution á la théorie générale de l'Etat, París, 1920, II, pp. 483 y ss. (trad. al castellano publicada por el Fondo de Cultura Económica, México, 1998); Burdeau, G., Traité de science politique, IV, París, 1983, cap. III; Mortati, C., Istituzioni di diritto pubblico, VIII ed., Padua, 1969, I, p. 64 y ss.; Barile, P., "Potere costituente", Novissimo digesto italiano, XIII, Turín, 1966; Dogliani, M., Potere costituente, Turín, 1986; Angiolini, V., Costituente e costituito nell'Italia repubblicana, Padua, 1995, pp. 74 y ss.; Carrió, G. R., Sobre los límites del lenguaje normativo, Buenos Aires, 1973, pp. 33 y ss.; Zagrebelsky, G., Manuale di diritto costituzionale, I, pp. 97 y ss.

22 La distinción viene desde Sieyés, Qu'est-ce que le Tiers Etat (1789).

23 Quisiera subrayar que, así entendido, el poder constituyente no es, en modo alguno, el fundamento axiológico de la Constitución, o algo por el estilo del género, sino simplemente el hecho del que la (primera) Constitución nace. De ello se sigue, entre otras cosas, que el poder constituyente no sobrevive al nacimiento de la (primera) Constitución, sino que desaparece en ella y con ella.

24 Kelsen, H., General Theory of Law and State, pp. 115 y ss.; id., Théorie pure du droit, pp. 264 y ss.

25 Con el corolario de que la primera Constitución ni es válida ni es inválida en el ámbito del ordenamiento que ella constituye. Sobre esto, Guastini, R., "Sur la validité de la constitution du point de vue du positivisme juridique", en M. Troper, L. Jaume (eds.), 1789 et l'invention de la constitution, Bruselas-París, 1994. 
Se puede convenir que el poder de reforma constitucional es un poder constituido (constituido por la Constitución existente), y que el poder de instauración constitucional sea por el contrario el poder constituyente. Ahora bien, ¿qué distingue la reforma constitucional, es decir la modificación de la Constitución existente, de la instauración constitucional, ${ }^{26}$ es decir de la emanación de una nueva Constitución?

\section{INSTAURACIÓN CONSTITUCIONAL VS. REFORMA DE LA CONSTITUCIÓN}

Esta pregunta admite (al menos) dos respuestas interesantes: cualquiera de ellas supone una diversa concepción de la Constitución (y de su criterio de identidad) e implica una diversa concepción del poder constituyente.

1. La concepción sustancial (o sustancialista). La primera respuesta suena grosso modo así: una Constitución es una totalidad coherente y conexa de valores ético-políticos. La identidad material (axiológica) de toda Constitución descansa precisamente en el conjunto de valores —o principios supremos- que la caracterizan y la distinguen de cualquier otra Constitución. ${ }^{27}$

Ahora bien, una cosa es reformar la Constitución existente (en sus normas de detalle) sin alterar la identidad material o axiológica; otra cuestión es modificar el "espíritu" de la Constitución existente, o sea alterar, perturbar o subvertir los valores ético-políticos que la caracterizan. $^{28}$

Una cosa es la simple reforma constitucional, otra -aunque sea enmascarada - es la instauración de una nueva Constitución. Una cosa es el ejercicio de un poder constituido (el poder de revisión), y otra es el ejercicio del poder constituyente.

26 Cfr. Romano, S., "L'instaurazione di fatto di un ordinamento costituzionale e la sua legittimazione”, en id., Lo Stato moderno e la sua crisi. Saggi di diritto costituzionale, Milán, 1969.

27 Schmitt, C., Dottrina della costituzione (1928), Milán, 1984, pp. 109 y ss. (trad. al castellano, Alianza Editorial, Madrid, 1992).

28 "La función constituyente se caracteriza por la libertad absoluta de producción, en comparación con la cual el radio más estrecho de la función de revisión constitucional, fundándose en la Constitución y por ello repitiendo el propio origen de la función constituyente, no puede tener un título para sustituirse a ésta sino que puede solamente actuar dentro del ámbito de acción que le ha sido asignado... La función constituyente infiere la propia superioridad frente a la función de revisión no tanto por su ilimitación originaria sino más bien en cuanto instrumento técnico de expresión de las elecciones y decisiones fundamentales a las que se asigna el nombre de Constitución en sentido material" (Cicconetti, S. M., La revisione della costituzione, pp. 229 y 231). 
Reforma e instauración constitucional se distinguen, entonces, no bajo un perfil formal - por el hecho de que una adviene en forma legal y otra de forma ilegal, extra ordinem - sino bajo el perfil sustancial: es una reforma toda modificación marginal, es instauración toda alteración aunque legal — de la identidad axiológica de la Constitución.

De lo anterior se sigue, entre otras cosas, que en ningún caso puede la reforma constitucional ser llevada hasta modificar los principios supremos de la Constitución existente. Tales principios son límites (lógicos) infranqueables para la reforma constitucional. ${ }^{29}$

2. La concepción formal (o formalista). La segunda respuesta suena grosso modo así: una Constitución no es más que un conjunto de normas. Ahora bien, un conjunto (cualquier tipo de conjunto) se identifica - extensionalmente - por la simple enumeración de los elementos que lo componen. $^{30}$

Se sigue, ante todo, que existen tres tipos posibles de reforma constitucional: a) la introducción de una norma nueva; b) la supresión de una norma preexistente; c) la sustitución de una norma preexistente (es decir la supresión de una norma vieja combinada con la introducción de una norma nueva). ${ }^{31}$ Pero se sigue también que, comúnmente, toda reforma constitucional comporta la modificación del conjunto preexistente, y la modificación de un conjunto da lugar a un conjunto diverso: diverso porque son diversos los elementos que lo componen.

Así, toda reforma constitucional —por más "marginal” que sea desde un punto de vista axiológico- produce una nueva Constitución. De modo que reforma constitucional e instauración constitucional son —desde un punto de vista wertfrei, avalorativo- cosas simplemente indistinguibles bajo un perfil sustancial. No resta entonces más que distinguir reforma e instauración sobre la base de elementos puramente formales.

Toda modificación constitucional realizada en forma legal — por más que pueda incidir profundamente sobre la Constitución existente- es mera reforma. Toda modificación realizada en forma ilegal — por más

29 Límites "lógicos", ya que una sedicente revisión constitucional que pretendiera alterar la identidad material de la Constitución se transformaría, por eso mismo, en instauración constitucional: es decir, por definición, no podría más llamarse "revisión constitucional".

30 Bulygin, E., Norme, validità, sistemi normativi, Turín, 1995, pp. 76 y ss.; Guastini, R., Distinguendo. Studi di teoria e metateoria del diritto, Turín, 1996, pp. 246 y ss.

31 Alchourrón, C. E., "Conflictos de normas y revisión de sistemas normativos”, en Alchourrón, C. E., Bulygin, E., Análisis lógico y derecho, Madrid, 1991, especialmente p. 301. 
marginal que pueda ser ese cambio- es instauración de una nueva Constitución. En suma: la modificación legal de la Constitución es ejercicio del poder constituido, mientras que su cambio ilegal es ejercicio del poder constituyente.

Desde este punto de vista, entre otras cosas, no tiene sentido hablar de límites lógicos a la reforma constitucional.

\section{LA LOCUCIÓN “CONSTITUCIÓN (EN SENTIDO) MATERIAL”}

Para concluir este trabajo, no se puede dejar de expresar algunas palabras para ilustrar los diversos significados de la locución "Constitución (en sentido) material", ${ }^{32}$ que recorre con frecuencia el lenguaje teórico y doctrinal (y también, para ser francos, el lenguaje político vulgar). ${ }^{33}$ Pues bien, de modo sucinto se puede decir esto:

En un primer sentido, se habla de Constitución material para referirse a las normas que, en cualquier ordenamiento, determinan la "forma de Estado" y la "forma de gobierno". 34

En un segundo sentido, se habla de Constitución material para referirse al conjunto de normas sobre la legislación, o más en general al conjunto de normas sobre las fuentes. ${ }^{35}$

En un tercer sentido, la misma expresión es utilizada para designar la "decisión política fundamental del titular del poder constituyente". 36

La "decisión política fundamental", a su vez, puede ser concebida: o como la decisión relativa a la forma de Estado, ${ }^{37}$ o como la decisión relativa al conjunto de los principios supremos que caracterizan a cualquier ordenamiento. ${ }^{38}$

32 Con la advertencia que el inventario que sigue es ciertamente incompleto.

33 A propósito: Mortati, C., La costituzione in senso materiale, Milán, 1940; id., "Costituzione (dottrine generali e costituzione della repubblica italiana)", Enciclopedia del diritto, XI, Milán, 1962; id., Istituzioni di diritto pubblico, I, 26 y ss. Cfr. además las obras citadas en la nota 3.

34 Cfr. Paladin, L., Diritto costituzionale, 25. Cfr. también Smend, R., Costituzione e diritto costituzionale, Milán, 1988, p. 147 (trad. al castellano, Centro de Estudios Constitucionales, Madrid, 1985).

35 Así, Kelsen, H. General Theory of Law and State, pp. 124 y ss., pp. 258 y ss.; id., Théorie pure du droit, pp. 299 y ss.

36 Schmitt, C., Dottrina della costituzione, p. 41.

37 Así parece concebirla Schmitt, C., Dottrina della costituzione, pp. 41 y ss.

38 Paladin, L., Diritto costituzionale, p. 22. 
En un cuarto sentido, se habla de Constitución material para referirse al régimen político vigente en un Estado. ${ }^{39}$

El régimen político, a su vez, es concebido: ${ }^{40}$ o como el conjunto de los "fines políticos en vista de los cuales las fuerzas dominantes inspiran la acción estatal" ; ${ }^{41}$ o bien como "el real arreglo y funcionamiento de las instituciones políticas en las varias fases históricas, al margen de cuanto prescriban las correspondientes cartas constitucionales". ${ }^{42}$

En un quinto sentido, en fin, la locución "Constitución material" es utilizada como sinónimo de "Constitución viva".

A su vez, la expresión "Constitución viva" denota — con un lenguaje sugestivo - el modo en que una determinada Constitución escrita es concretamente interpretada y actuada en la realidad política. Por lo que se refiere a la interpretación, es bastante obvio que cualquier texto constitucional es susceptible de interpretaciones no sólo sincrónicamente diversas, sino (sobre todo) diacrónicamente cambiantes. Por lo que respecta a la actuación, habría quizá que aclarar que un texto constitucional (no diversamente, por lo demás, de lo que sucede con cualquier otro texto normativo) puede permanecer inactuado. Se puede crear un problema de "actuación" constitucional, y correlativamente se abre la posibilidad de que la Constitución quede "inactuada" en al menos dos clases de normas.

Por un lado, las eventuales normas programáticas, dirigidas al legislador. Muchas Constituciones contemporáneas contienen normas de este tipo.

Por otro lado, las eventuales normas — como se suele decir- "de eficacia diferida", es decir todas aquellas normas que no pueden adquirir eficacia sin la previa creación de otras normas, las cuales son, por ello, condición necesaria de eficacia. ${ }^{4}$

39 Cfr. por ejemplo Barile, P., La costituzione come norma giuridica, Florencia, 1951; A. M. Sandulli, "Fonti del diritto", Novissimo digesto italiano, VII, Turín, 1961, p, 527.

40 Cfr. Cicconetti, S. M., La revisione della costituzione, p. 232.

41 Así, Sorrentino, F., Le fonti del diritto, IV ed., Génova, 1997, p. 29 (con importantes observaciones críticas). Este modo de utilizar la expresión en examen puede remitirse a Mortati, C., $L a$ costituzione in senso materiale.

42 Paladin, L., Le fonti del diritto italiano, Bolonia, 1986, p. 127.

43 La falta de una norma que sea condición necesaria de eficacia de otra norma constituye lo que se acostumbra llamar una "laguna técnica". 